\title{
Prevalence and impact of WHO group 3 pulmonary hypertension in advanced idiopathic nonspecific interstitial pneumonia
}

\author{
To the Editor:
}

Pulmonary hypertension $(\mathrm{PH})$ is associated with impaired quality of life, worsened functional status and increased mortality in idiopathic pulmonary fibrosis (IPF) [1]. The prevalence and clinical impact in other idiopathic interstitial pneumonias (IIPs) has not previously been reported. Given the prognostic significance of $\mathrm{PH}$ in IPF, we sought to determine the prevalence and severity of World Health Organization (WHO) group $3 \mathrm{PH}$ in a cohort of patients with biopsy-proven idiopathic nonspecific interstitial pneumonia (NSIP). We recognised that the prevalence of $\mathrm{PH}$ in this population of patients with advanced NSIP would be an overestimation of the NSIP population at large, but felt that mandating surgical lung biopsy and right heart catheterisation (RHC) were the only way to ensure accurate diagnosis of NSIP and $\mathrm{PH}$, respectively.

A retrospective review of patients with idiopathic NSIP diagnosed between 2002 and 2016 at our centre was performed. All cases were reviewed at our multidisciplinary pulmonary pathology conference. Connective tissue disease (CTD) was rigorously excluded in all patients and RHC was required for confirmation of PH. RHCs are not performed standardly on all patients in our clinic, and would have been performed as part of lung transplant evaluation or to confirm $\mathrm{PH}$ suggested by clinical parameters or echocardiographic data. $\mathrm{PH}$ was defined as a resting mean pulmonary artery pressure (mPAP) $\geqslant 25 \mathrm{mmHg}$ and severe $\mathrm{PH}$ as $\mathrm{mPAP} \geqslant 35 \mathrm{mmHg}$. Patients with a pulmonary capillary wedge pressure $>15 \mathrm{mmHg}$ were excluded from the cohort as they were suspected to have left-heart disease associated (WHO group 2) $\mathrm{PH}$. Chronic thromboembolic disease was excluded in all patients with ventilation/perfusion scanning. The primary endpoints of this study were the prevalence and impact of WHO Group $3 \mathrm{PH}$ on patient outcomes in idiopathic NSIP. Secondary outcomes included the correlation of forced vital capacity (FVC) and mPAP in idiopathic NSIP patients, as well as the association between other demographics and $\mathrm{PH}$ in this population.

Review of records from our multidisciplinary pulmonary pathology meeting identified 95 potential patients with biopsy proven NSIP. Of these patients, 35 met criteria for inclusion in the study.

11 (31.4\%) out of 35 patients had WHO group $3 \mathrm{PH}$. The mean \pm SD mPAP for those with and without WHO group $3 \mathrm{PH}$ were $32.0 \pm 10.3 \mathrm{mmHg}$ and $18.1 \pm 3.1 \mathrm{mmHg}$, respectively. Of the 35 patients in the study, seven (20\%) patients had a mPAP of $25-30 \mathrm{mmHg}$, two $(5.7 \%)$ had a mPAP of $31-34 \mathrm{mmHg}$ and two $(5.7 \%)$ had a mPAP $\geqslant 35 \mathrm{mmHg}$. Table 1 details the characteristics of the patients with $\mathrm{PH}$ versus those without. Per cent predicted FVC was similar between the WHO group $3 \mathrm{PH}$ and no PH cohorts, with $\mathrm{r}=-0.12$ suggesting poor correlation between FVC per cent predicted and $\operatorname{mPAP}(\mathrm{p}=0.48)$.

Median transplant-free survival was significantly lower in patients with WHO group $3 \mathrm{PH}$ at 17.6 months as compared to 47.9 months in the cohort without $\mathrm{PH}(\mathrm{p}=0.05)$. Severe $\mathrm{PH}$ (mPAP $\geqslant 35 \mathrm{mmHg}$ or mPAP $\geqslant 25 \mathrm{mmHg}$ with a low cardiac index) was only seen in two (5.7\%) patients, both of whom expired without transplant, with an average adjusted survival time of 12.6 months. Both of these patients also suffered from severe functional impairment with a mean 6-min walk test distance of only 137.2 and $129 \mathrm{~m}$, respectively.

@ERSpublications

Group 3 pulmonary hypertension commonly complicates idiopathic NSIP and is associated with adverse outcomes http://ow.ly/jsa930jMaSD

Cite this article as: King CS, Brown AW, Shlobin OA, et al. Prevalence and impact of WHO group 3 pulmonary hypertension in advanced idiopathic nonspecific interstitial pneumonia. Eur Respir J 2018; 52 : 1800545 [https://doi.org/10.1183/13993003.00545-2018]. 
TABLE 1 Baseline characteristics of idiopathic nonspecific interstitial pneumonia (NSIP) patients stratified by the presence of group 3 pulmonary hypertension

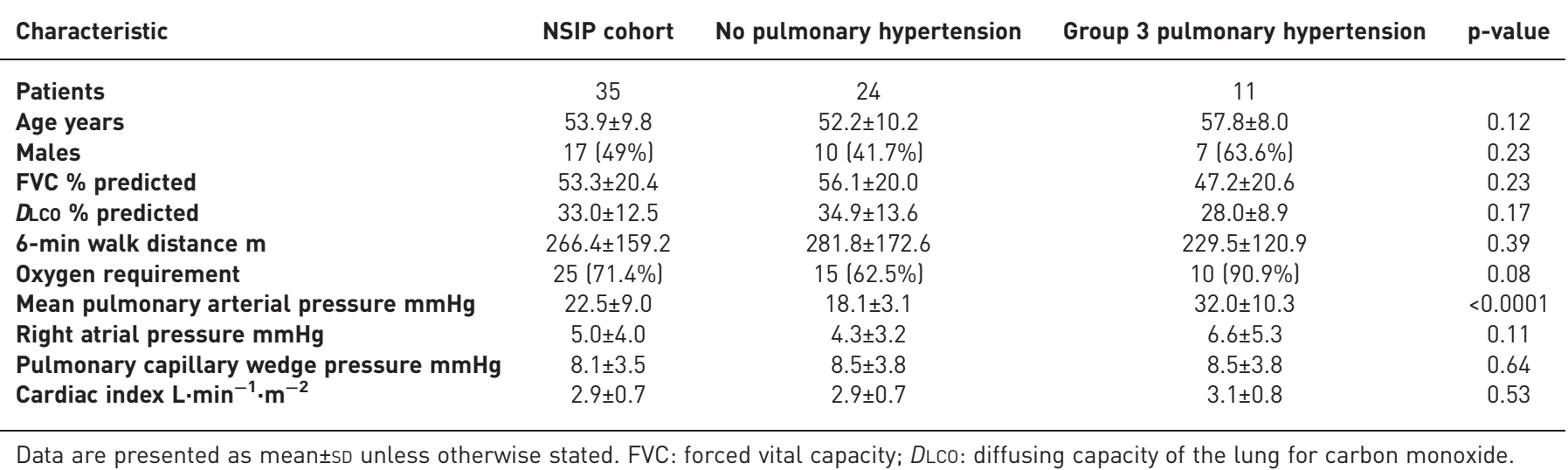

Data are presented as mean \pm SD unless otherwise stated. FVC: forced vital capacity; $D$ Lco: diffusing capacity of the lung for carbon monoxide.

A competing risk analysis was performed to determine if the relationship between WHO group $3 \mathrm{PH}$ and survival would persist after taking into account lung transplant as a competing event. The analysis confirmed that the presence of WHO group $3 \mathrm{PH}$ was associated with an increased risk of death even after considering transplant as a competing risk (hazard ratio 3.73, 95\% CI 1.32-10.55; $\mathrm{p}=0.013$ ). Additionally, a multivariate competing risk analysis found WHO group $3 \mathrm{PH}$ was still associated with an increased risk of death after adjusting for age and FVC per cent predicted.

We found that WHO group $3 \mathrm{PH}$ was present in approximately one-third (31.4\%) of idiopathic NSIP patients who underwent RHC. To our knowledge, this is the first and only study to rigorously evaluate $\mathrm{PH}$ in NSIP. In fact, no prior study has reported on the prevalence of PH in any IIP other than IPF. We feel our study has a number of strengths, including the strict inclusion criteria used to ensure that only idiopathic NSIP patients were included (need for surgical lung biopsy, extensive CTD serological evaluation and review by multidisciplinary pulmonary pathology committee), confirmation of WHO group $3 \mathrm{PH}$ by RHC and long-term follow-up.

The major finding from our study was the significant reduction in transplant-free survival seen in idiopathic NSIP patients with WHO group $3 \mathrm{PH}$. Median transplant-free survival was over 30 months shorter in patients with WHO group $3 \mathrm{PH}$, at a mere 17.6 months. The association of WHO group $3 \mathrm{PH}$ with increased mortality remained, even after adjusting for age and FVC \% predicted, and treating lung transplant as a competing risk. The association between the development of $\mathrm{PH}$ is consistent with what has been demonstrated in the IPF population, where PH serves as a powerful predictor of mortality $[1,2]$. Indeed, it appears that once PH develops in NSIP and IPF, outcomes are quite similar, suggesting perhaps that $\mathrm{PH}$ then becomes the major driver of patients' subsequent clinical course. Given this association, it is prudent for clinicians to screen for $\mathrm{PH}$ and consider lung transplant listing in NSIP patients who develop complicating $\mathrm{PH}$.

The prevalence of $\mathrm{PH}$ in this idiopathic NSIP population is likely to be an overestimate of what would be seen in a less select, general pulmonary practice population for several reasons. Since our clinic specialises in advanced lung disease and lung transplantation, there is inherent referral bias and the study population represents a more advanced subset of idiopathic NSIP patients, as their mean FVC was 53.3\% predicted. In addition, all 35 patients in our study had mixed cellular and fibrotic or fibrotic NSIP pathology, and thus may represent a more treatment-refractory subset of idiopathic NSIP. Lastly, the RHC requirement for inclusion in the study makes the pre-test probability of PH higher as the indication for RHC would typically have been clinical or echocardiographic suspicion of $\mathrm{PH}$, or as part of a pre-transplant evaluation. Regardless, given the paucity of data on the prevalence of PH in NSIP, we feel these data are of value to clinicians, particularly those who practice in a clinic with a similar patient population.

Although not our original intention, our study also contributes to the existing literature on survival in idiopathic NSIP. Travis et al. [3] reported a 90\% 5-year survival for idiopathic fibrotic NSIP. Transplant-free survival in our cohort, even in those without $\mathrm{PH}$, was $<50 \%$ at 5 years. Ours most likely reflects a more advanced population, as noted by the moderately severe mean FVC, and its composition of only mixed cellular and fibrotic or fibrotic NSIP patients. In addition, Travis et al. [3] exclusively evaluated death in their survival analysis, not lung transplantation, whereas our study takes into account 
both outcomes, which likely contributes to the shorter transplant-free survival. Lastly, the methods used to ascertain patient survival differ between the two studies. The Travis et al. [3] study relied upon review of medical records and contact with the referring physician, which may have underestimated the incidence of death compared to our use of the Social Security Death Index, which may be more accurate as patients relocate or are lost to follow-up. Nonetheless, outcomes in our cohort are notably worse than prior reports of NSIP.

In conclusion, we have demonstrated that $\mathrm{PH}$ commonly complicates the course of idiopathic fibrotic NSIP. Development of $\mathrm{PH}$ in this population is associated with markedly reduced transplant-free survival. Indeed, PH related to NSIP appears to have similar prognostic implications to what has been reported in IPF-PH, supporting the current concept of including both groups in the same clinical trials of therapy.

Christopher S. King ${ }^{1}$, A. Whitney Brown ${ }^{1}$, Oksana A. Shlobin ${ }^{1}$, Nargues Weir ${ }^{1}$, Matthew Libre ${ }^{2}$, Domingo Franco-Palacios ${ }^{3}$, Shahzad Ahmad ${ }^{4}$ and Steven D. Nathan ${ }^{1}$

${ }^{1}$ Inova Fairfax Hospital Advanced Lung Disease and Lung Transplant Program, Inova Fairfax Hospital, Falls Church, VA, USA. ${ }^{2}$ Dartmouth University, Haven, NH, USA. ${ }^{3}$ Division of Pulmonary/Critical Care, Carilion Clinic, Roanoke, VA, USA. ${ }^{4}$ Center for Advanced Lung Disease, Stanford University Medical Center, Stanford, CA, USA.

Correspondence: Christopher S. King, Inova Fairfax Hospital, Advanced Lung Disease and Transplant Clinic, 3300 Gallows Road, Falls Church, VA 22042, USA. E-mail: Christopher.king@inova.org

Received: Jan 232018 | Accepted after revision: April 052018

Conflict of interest: C.S. King has received personal fees from Boehringer Ingelheim and from the France Foundation for the PILOT program, outside the submitted work. O.A. Shlobin has acted as a consultant, advisory board member and speaker for Bayer, United Therapeutics and Actelion. S.D. Nathan is a consultant for, has received research funding from and is on the speakers bureau for Boerhinger-Ingelheim, Gilead Sciences, Roche-Genentech, Bayer Pharmaceuticals and United Therapeutics. He is also a consultant for Bellerophon.

\section{References}

1 Lettieri CJ, Nathan SD, Barnett SD, et al. Prevalence and outcomes of pulmonary arterial hypertension in advanced idiopathic pulmonary fibrosis. Chest 2006; 129: 746-752.

2 Nadrous HF, Pellikka PA, Krowka MJ, et al. Pulmonary hypertension in patients with idiopathic pulmonary fibrosis. Chest 2005; 128: 2393-2399.

3 Travis WD, Matsui K, Moss J, et al. Idiopathic nonspecific interstitial pneumonia: prognostic significance of cellular and fibrosing patterns: survival comparison with usual interstitial pneumonia and desquamative interstitial pneumonia. Am J Surg Pathol 2000; 24: 19-33. 